\title{
Investigation report and countermeasures analysis of express package pollution
}

\author{
Jiaqian Dong \\ School of Electrical \& Electronic Engineering, North China Electric Power University, Baoding, \\ 071003 China
}

2827947422@qq.com

Keywords: express package pollution; questionnaire; statistics analysis; strategy.

\begin{abstract}
With the gradual population of e-commerce and online shopping, together with the rapid development of logistics industry, the pollution caused by express packages has intensified. In order to solve the pollution of express packages, this paper employed qualitative and quantitative research methods of questionnaires and statistic analysis, and finally put forward solutions based on college students. This report included two main aspects. The one was to do investigations, aiming at pinpointing the problems we had to solve under the circumstance of express package pollution. The other was to think of strategies to meet the current requirements of energy saving and to achieve sustainable development.
\end{abstract}

\section{Introduction}

Affected by the e-commerce and online shopping, the express industry has been developing by explosive growth recent years. However, plenty of litter from the express packaging is also produced with that and it is still rapidly increasing. The total business of the express companies that has covered the whole country had reached 9.19 billion deliveries and at the same time, billions of package waste such as the paper, plastic waste was produced. It not only causes wasting of resources, but also the pollution to the environment. Now the public begin to pay attention to that gradually. At present the college students have become the main group in online shopping. The most of them would throw the express package away and only a small percentage of them would recycle. So we did the questioning reports aiming at college students and couriers and found the problems and the countermeasures according the analysis.

This investment is based on two questioning reports adding unstructured interview, document research and so on. About 950 questioning papers were put on the net and the effective questioning papers were about 920 reaching the $96.48 \%$ effective rate.

\section{Conclusion:}

Many of the college students have the habit of online shopping. The quality is large and is still increasing.

1. The value of retail sales of the online dealers whose business is above the limit is 82.5 billion yuan in 2014 first quarter and the year-on-year growth is $51.7 \%$ according to the data. It is invested that $39.45 \%$ of the interviewee often shop online, $4.59 \%$ of them occasionally shop online among the college students invested. And $88.07 \%$ of them are under five express packages and $11.98 \%$ are about 6 to 15 express packages.

2. The main way people deal with the express package is throwing for there are no regular recycling systems.

Because there are not the concerning rules about recycling express package in China, people have the low sense of express package recycling. So when asked how to deal with the express package, $77.98 \%$ of the interviewees throw them away directly and only $6.42 \%$ of them will recycle the express package. 
3. Express delivery industry will be at a relatively high speed of development in the next few years.

According to the courier survey volume, 71.58 percent of the couriers deem that the growth trend over the past few years is very fast, or at least relatively fast. $76.84 \%$ of the express couriers deem that the next few years the growth will be even faster. Thus, according to the above findings, it can be inferred that express waste generation will show a growing trend in the future, and will generate a lot of courier garbage.

4. Consumers and couriers have been aware of the enormous courier waste, which is harm to the environment and the human body.

In awareness of the express garbage, $67.89 \%$ of consumers believe courier garbage is becoming more. And $74.74 \%$ of the couriers suggest that the online shopping area produces a huge amount of garbage. In the awareness of the hazards the courier industry brings to the environment, air and human, $27.86 \%$ of consumers believe that transparent tape, polyethylene courier bags and others are difficult to degrade and landfill or incinerating may contaminate soil or water resources. $23.53 \%$ of consumers believe that the excessive use of plastic bags may make white pollution more serious. 28.02\% of the couriers think that the excessive packaging will bring certain economic losses. It can be found that whether consumers or express couriers have recognized that the waste brings great hazards to the environment and human body.

5. Most consumers have indicated that participate actively in the recovery process of express garbage, and setting appropriate incentives may attract more consumers to participate.

According to survey results, $37.61 \%$ of consumers say they are willing to participate actively in and call on others to join the courier waste recycling activities. $59.63 \%$ of consumers say they would participate in as long as the time is right. Specific participation measures include: Courier packaging recycling (28.97\%), the implementation of paid use of courier package (16.67\%) and collecting the packaging for other uses (20.24\%). In addition, under certain incentives, consumers will be greatly stimulated by the recovery enthusiasm. Therefore, when we are determining the courier garbage solutions, the introduction of certain incentive policy can facilitate the smooth development of garbage recycling.

Table 1. Incentives questionnaire analysis

\begin{tabular}{ccc}
\hline category & quantity & ratio \\
\hline Credit for 3White bags/boxes & 335 & $61.47 \%$ \\
Credit for 5White bags/boxes & 130 & $23.85 \%$ \\
Credit for 10White bags/boxes & 45 & $8.26 \%$ \\
No participation & 35 & $6.42 \%$ \\
Total & 545 & 100.0 \\
\hline
\end{tabular}

6. The difficulty analysis for the promotion of environmentally-friendly packaging, standard boxes and others.

According to the survey, 34.13 percent of consumers say express industry should be encouraged to promote green biodegradable bags / boxes. And $83.16 \%$ of the couriers deem that it is necessary to promote the use of standard containers. Therefore, we can be find that these two solutions of courier garbage are feasible. However, a significant number of consumers expressed resentment against the compensation for using courier packaging.

Based on the above data to understand the status of express packaging pollution issues, this paper proposes the following main sub-measures:

1. Consumers

(1) Awareness. Consumers should improve their sense of social responsibility, human rights awareness and environmental awareness. They should consciously buy green packaging, with little or no disposable packaging, resisting excessive packaging, packaging fraud and packaging contaminated.

(2) Protection. In the process of dismantling the box, consumers should do carefully, to avoid damage to packaging and to maximize the protection of the good status quo of boxes. 
(3) Re-use. We can put exquisite cartoon confetti on the surface of the waste delivery cartons, and to separate with a small box in the middle, converting it into a storage box. The relatively hard texture rectangular box can be modified into a pen case. The special express black plastic bags, if cut opened close to one end, can be used as disposable garbage bags, as well as preventing waste residue and water from leaking out.

2. Electricity suppliers

(1) Packaging material. Preference is given to those low energy consumption, low pollution, and reusable, easy-recycling and biodegradable corruption materials. At the same time, develop new biodegradable, photodegradable, water soluble and edible packaging materials.

(2) Packaging Design. Under the premise of reductions, design those environmentally friendly, economical, pleasant, artistic, and security products.

(3)Produce. Pay attention to the progress of packaging and packaging technology improvements. Use the advanced packaging technology and packaging standards to carry out clean production.

(4) Recovery and recycling. Pay attention to the importance of packaging waste recovery and recycling, saving energy and resources.

\section{Company}

Companies should establish the values of caring for the environment, and promote the coordinated development of material civilization and ecological civilization. Improve the quality of the paper and reduce paper weight. Use moderate materials in the packaging process as much as possible, and put the thoughts of consuming resources and ecological environment into the design concept.

(2) To achieve a good balance between the relationship of economic interests and social responsibilities, and try to use biodegradable materials or promote the recycling of packaging. We can also use a standardized packaging design. For the Standardized packaging design, package size should meet the requirements of adapting to the tools like pallets, containers, and transport carriers in the transport system, maximizing the utilization of the means of transport.

(3) Establish specialized packaging recovery mechanism. We can simultaneously consider aspects of the couriers, fixed communities and incentives, to establish a sound express packaging recycling mechanism.

\section{Government}

(1)Legally clearly define over-packaging, packaging fraud and packaging contamination. For over-packaging, we should set limits from the aspects of the volume of packaging material, the gap between packaging and product, packaging layers and the proportion of packaging cost and product value. For packaging fraud, we should set limits from whether the characteristics of the product inside the packaging are consistent with the instructions on the packaging. For packaging contamination, we should set limits from the type and quantity of emissions, to implement a unified criteria and standards.

(2) Formulate "green standards" in express delivery industry economically, and encourage relevant research institutions to carry out the integration of resources, to develop a well-functioning, affordable, and environmentally friendly green standard design packages. Develop science and technology innovation, and manage scientifically from aspects of product packaging design, material selection, product recovery and others. Select harmless type of environmentally friendly materials, and use advanced technology to improve the packaging recovery and utilization rates.

\section{References}

[1] Jianing Zhang, Fang Liu. The thinking of low-carbon express packaging design[J].Packaging engineering, 2014,04:82-85.

[2] Yingyan Chen. Innovative design of express package under the concept of sustainable development[J].Packaging engineering, 2014,24:10-12+26.

[3] What are the difficulties of courier packaging recycling[J].China packaging,2015,03:61-65.

[4]Cheng Wang. Green courier package suitable for "online shopping"[J]. China packaging, 2010,07:52-55. 
[5] Dongmei An,Xin Han. Effective measures to improve the delivery package of normalization[J]. Value engineering,2013,06:322-323. 